\title{
UNIVERSITY OF LUND RADIOCARBON DATES XIX
}

\author{
SÖREN HÅKANSSON
}

Radiocarbon Dating Laboratory, Department of Quaternary Geology

University of Lund, Sweden

\section{INTRODUCTION}

Most of the ${ }^{14} \mathrm{C}$ measurements reported here were made between October 1984 and October 1985. Equipment, measurement, and treatment of samples are as reported previously (R, 1968, v 10, p 36-37; 1976, v 18 , p 290; 1980, v 22, p 1045) except that 23 separate GM-counters used as guard counters were replaced by a single iron multiwire ring-counter filled with $90 \mathrm{Ar} / 10 \mathrm{CH}_{4}$ to ca $130 \mathrm{kPa}$ (ca $\left.1.3 \mathrm{~atm}\right)$. Background counts were reduced by ca $12 \%$ due to this change. High voltage supply for main detectors was replaced as well.

Age calculations are based on a contemporary value equal to $95 \%$ of the activity of NBS oxalic acid standard (No. 4990A) and on the conventional half-life for ${ }^{14} \mathrm{C}$ of $5568 \mathrm{yr}$. Results are reported in years before 1950 (years BP). Errors quoted with the dates are based on counting statistics alone and are equivalent to \pm 1 standard deviation $( \pm \sigma)$.

Corrections for deviations from $\delta^{13} \mathrm{C}=-25.0 \%$ in the PDB scale are applied for almost all samples; also for marine shells. The apparent age for marine material due to the reservoir effect must be subtracted from our dates on such samples.

The remark "undersized; diluted," in Comments means the sample did not produce enough $\mathrm{CO}_{2}$ to fill the counter to normal pressure and "dead" $\mathrm{CO}_{2}$ from anthracite was introduced to make up the pressure. "\% sample", indicates amount of $\mathrm{CO}_{2}$ derived from the sample present in the diluted counting gas; the rest is "dead" $\mathrm{CO}_{2}$. Organic carbon content reported for bone samples is calculated from yield of $\mathrm{CO}_{2}$ by combustion of gelatine remaining after treatment. Organic carbon lost during treatment is not included in calculated percentage.

The description of each sample is based on information provided by the submitter.

\section{ACKNOWLEDGMENTS}

The author thanks Kerstin Lundahl for sample preparation and routine operation of the dating equipment, and Owe Gustafsson, Dept of Marine Geology, University of Göteborg, for mass-spectrometric determination of $\delta^{13} \mathrm{C}$. Special thanks are due the staff of the engineering workshop at the Chemical Centre in Lund for manufacturing the basic parts of the new ring-counter.

\section{GEOLOGIC SAMPLES}

Sweden

\section{Subfossil Pine Series I}

Subfossil wood (Pinus silvestris) from Bergfors Lakes $\left(68^{\circ} 10.2^{\prime} \mathrm{N}, 19^{\circ}\right.$ $5\left(0.2^{\prime} \mathrm{F}\right)$ and Abisko Lake ( $\left.68^{\circ} 22.2^{\prime} \mathrm{N}, 19^{\circ} 2.8^{\prime} \mathrm{E}\right), \mathrm{S}$ of Torncträsk, I appland. 
Coll 1983 and subm by T Bartholin, Lab Wood Anatomy and Dendrochronol, Dept Quaternary Geol, Univ Lund. Trees were found on lake bottom near shore. Dated to help connect different parts of floating pine chronologies for N Sweden. All samples except Lu-2253 and -2254 were charred in nitrogen atmosphere before burning.

\section{Lu-2253. Bergfors Lake 1, Sample 220380 \\ $4030 \pm 60$}

Wood from annual rings No. 100 to 147 . Comment: no pretreatment.

\section{Lu-2254. Bergfors Lake 1, Sample $221170 \quad \delta^{13} C=-26.2^{\%} \% 0$ \\ Wood from annual rings No. 5 to 30 . Comment: no pretreatment.}

\section{Lu-2298. Bergfors Lake 1, Sample 22028 \\ $3570 \pm 60$ \\ $\delta^{13} C=-27.5 \%$}

Wood from annual rings No. 180 to 352 . File No. I 14 M. Comment: pretreated with $\mathrm{NaOH}$ and $\mathrm{HCl}$.

Lu-2299. Bergfors Lake 1, Sample 22029

$$
\begin{array}{r}
\mathbf{2 9 1 0} \pm \mathbf{5 0} \\
\delta^{13} C=-26.4 \%
\end{array}
$$

Wood from annual rings No. 100 to 220 . File No. I 7 M. Comment: pretreated with $\mathrm{NaOH}$ and $\mathrm{HCl}$.

Lu-2300. Bergfors Lake 1, Sample 22040

$$
\begin{array}{r}
\mathbf{2 7 5 0} \pm \mathbf{5 0} \\
\delta^{13} C=-27.6 \%
\end{array}
$$

Wood from annual rings No. 1 to 20. File No. I 1 M. Comment: pretreated with $\mathrm{HCl}$.

Lu-2301. Bergfors Lake 1, Sample 22072

$$
\begin{array}{r}
\mathbf{2 0 0 0} \pm \mathbf{5 0} \\
\delta^{13} C=-26.9^{0} \% 0
\end{array}
$$

Wood from annual rings No. 1 to 25. File No. I 4 M. Comment: no pretreatment.

Lu-2302. Bergfors Lake 1, Sample 22095

$$
\begin{array}{r}
\mathbf{3 1 5 0} \pm \mathbf{5 0} \\
\delta^{13} C=-26.5 \% 0
\end{array}
$$

Wood from annual rings No. 50 to 80 . File No I 3 M. Comment: pretreated with $\mathrm{HCl}$ and $\mathrm{NaOH}$.

Lu-2303. Bergfors Lake 4, Sample 22137

$$
\begin{array}{r}
\mathbf{3 5 4 0} \pm \mathbf{6 0} \\
\delta^{13} C=-26.3 \%
\end{array}
$$

Wood from annual rings No. 70 to 140 . File No. I 13 M. Comment: pretreated with $\mathrm{NaOH}$ and $\mathrm{HCl}$.

Lu-2304. Bergfors Lake 5, Sample 22173

$1760 \pm 50$

Wood from annual rings No. 1 to 20 . File No. I 8 M. Comment: pretreated with $\mathrm{HCl}$. 
Lu-2305. Bergfors Lake 5, Sample 22176

$3390 \pm 60$ treated with HCl.

Lu-2306. Abisko Lake 4, Sample 25045

$6050 \pm 70$

Wood from annual rings No. 1 to 22. File No. I 12 M. Comment: pretreated with $\mathrm{HCl}$ and $\mathrm{NaOH}$.

\section{Håkulls Mosse series (IV)}

Wood fragments from Håkulls Mosse on hill ridge Kullaberg, NW Scania $\left(56^{\circ} 17^{\prime} \mathrm{N}, 12^{\circ} 31^{\prime} \mathrm{E}\right)$, alt ca $125 \mathrm{~m}$. Coll 1983 and subm by G Lemdahl, Dept Quaternary Geol, Univ Lund. Samples taken with piston corer, diam $10 \mathrm{~cm}$. Coring Point B8:6. For other dates from Håkulls Mosse, see R, 1978, v 20, p 416-417; 1980, v 22, p 1049-1050; 1984, v 26, p 394-395.

Lu-2354. Håkulls Mosse, H 1

$11,440 \pm 100$

Unid. small wood fragments, 401 to $405 \mathrm{~cm}$ below surface. Farly part of Aller $\phi \mathrm{d}$ pollen zone according to pollen analysis. Comment: no pretreatment.

\section{Lu-2365. Håkulls Mosse, G-I}

$11,480 \pm 100$

Wood (Salix sp) id by T Bartholin, 391 to $397 \mathrm{~cm}$ below su part of Aller $\phi \mathrm{d}$ pollen zone. Comment: pretreated with $\mathrm{HCl}$ and $\mathrm{NaOH}$.

\section{Åkhultmyren series}

Peat from Åkhultmyren, $3 \mathrm{~km}$ SSW of Aneboda church, Småland $\left(57^{\circ}\right.$ $06^{\prime} \mathrm{N}, 14^{\circ} 32^{\prime} \mathrm{E}$ ). Coll 1983 and subm by G Svensson, Dept Plant Ecol, Univ Lund. Previous study of Åkhultsmyren pub by N Malmer (1962). Most samples are from transition bog/mire peat. Depths $(\mathrm{cm})$ refer to mire surface. Von Post humification is given in H1-10 scale. All samples pretreated with $\mathrm{HCl}$ and charred in nitrogen atmosphere before burning.

$$
\text { Lu-2389. Åkhultsmyren I, } 86 \text { to } 90 \mathrm{~cm} \quad \delta^{13} C=\begin{array}{r}
\mathbf{8 2 0} \pm \mathbf{4 5} \\
-26.4 \%
\end{array}
$$

Sphagnum cuspidata-Cyperaceae peat, H 5-6, Coring Point Å 1 .

Lu-2390. Åkhultsmyren II, 122 to $126 \mathrm{~cm}$

Sphagnum cuspidata peat, H 4, Coring Point $\AA 1$.

Lu-2391. Åkhultsmyren III, 97 to $100 \mathrm{~cm}$

Sphagnum peat, H5-6, Coring Point $\AA 2$.
$1110 \pm 45$

$\delta^{13} C=-26.7 \%$

$670 \pm 45$

$\delta^{13} C=-26.3 \%$ 


\section{Lu-2392. Åkhultsmyren IV, 117 to $120 \mathrm{~cm}$}

$1150 \pm 60$

$\delta^{13} \mathrm{C}=-22.9 \%$

Sphagnum cuspidata peat, H 3, Coring Point Å 3. Comment: sample undersized; diluted; $72 \%$ sample.

\section{Lu-2393. Åkhultsmyren V, 70 to $73 \mathrm{~cm}$}

$970 \pm 45$

Sphagnum acutifolia peat, H 3, Coring Point Å 4 .

\section{Lu-2394. Åkhultsmyren VI, 134 to $137 \mathrm{~cm}$}

$$
\delta^{13} C=-25.8 \%
$$

Sphagnum cuspidata peat, H 5, Coring Point Å 4. Comment: sample undersized; diluted; $92 \%$ sample.

\section{Eastern Småland series (II)}

Sediment from lakes Flågöl $\left(57^{\circ} 13^{\prime} \mathrm{N}, 16^{\circ} 20^{\prime} \mathrm{E}\right)$, Hörnegöl $\left(57^{\circ} 10^{\prime} \mathrm{N}\right.$, $\left.16^{\circ} 11^{\prime} \mathrm{E}\right)$, Djupeträsk ( $\left.57^{\circ} 16^{\prime} \mathrm{N}, 16^{\circ} 22^{\prime} \mathrm{E}\right)$, Bastgöl $\left(57^{\circ} 13^{\prime} \mathrm{N}, 16^{\circ} 19^{\prime} \mathrm{E}\right)$, and Skvarran (57 $\left.12^{\prime} \mathrm{N}, 16^{\circ} 09^{\prime} \mathrm{E}\right)$, Kalmar län, E Småland. Coll 1984 and 1985 and subm by N-O Svensson, Dept Quaternary Geol, Univ Lund. Dated as complement to Eastern Småland series (R, 1986, v 28, p $141-$ 143). Depths (cm) refer to sediment surface. Samples were taken with Russian-type corer, diam $10 \mathrm{~cm}$. All samples pretreated with HC1. Organic carbon content given in Comments is calculated from final yield of $\mathrm{CO}_{2}$ and based on amount of material remaining after pretreatment. Loss of organic carbon during processing of sample and non-proportional loss during pretreatment may result in somewhat lower values than original ones.

\section{Flågöl}

Lu-2415. Flågöl 1, 781.5 to $784 \mathrm{~cm}$

$9570 \pm 90$

Clay gyttja. Comment: organic carbon content: ca $8 \%$.

Lu-2416. Flågöl 2, 750 to $753 \mathrm{~cm}$

$9360 \pm 90$

Clay gyttja. Comment: organic carbon content: ca 5\%.

\section{Hörnegöl}

Lu-2417. Hörnegöl 1, 465 to $468 \mathrm{~cm}$

$10,920 \pm 100$

Muddy clay. Comment: organic carbon content: ca $2 \%$.

Lu-2418. Hörnegöl 2, 456 to $459 \mathrm{~cm}$

$10,380 \pm 90$

Clay gyttja. Comment: organic carbon content: ca $3 \%$.

$$
\delta^{13} C=-24.1 \%
$$


Lu-2419. Hörnegöl 3, 449.5 to $452 \mathrm{~cm}$

$9880 \pm 90$

Clay gyttja. Comment: organic carbon content: ca $7 \%$.

\section{Djupeträsk}

Lu-2479. Djupeträsk 1, 551 to $559.5 \mathrm{~cm}$

Clay gyttja. Pollen zone boundary BO 1/BO 2. Comment: organic carbon content: ca $2.5 \%$.

Lu-2480. Djupeträsk 2, 488 to $492.5 \mathrm{~cm}$.

$8440 \pm 80$

Claycy $\quad \delta^{13} C=-29.5 \%$ $6 \%$.

Clayey gyttja. Pollen zone BO 2. Comment: organic carbon content: ca

\section{Bastgöl}

Lu-2481. Bastgöl 3, 371.5 to $374.5 \mathrm{~cm}$

$$
\begin{array}{r}
\mathbf{9 4 7 0} \pm \mathbf{9 0} \\
\delta^{13} \mathrm{C}=-24.5 \% 0
\end{array}
$$
ca $12 \%$.

Clay gyttja. Prcboreal pollen zone. Comment: organic carbon content:

\section{Skvarran}

\section{Lu-2482. Skvarran $3,416.5$ to $419.5 \mathrm{~cm}$}

$10,320 \pm 90$
$\delta^{13} C=-22.20$

Clay gyttja. Younger Dryas pollen zone. Comment: organic carbon content: ca $5 \%$.

\section{Lu-2483. Skvarran 5, 399.5 to $402.5 \mathrm{~cm}$}

$$
\begin{array}{r}
\mathbf{1 0 , 0 8 0} \pm \mathbf{9 0} \\
\delta^{13} C=-21.6 \%
\end{array}
$$

Fine detritus gyttja. Preboreal pollen zone. Comment: organic carbon content: ca $9 \%$.

\section{Kräckelbäcken series}

Peat, sndiment, and wood from aapamire $3 \mathrm{~km} \mathrm{SW}$ of Kräckelbäcken, Dalarna $\left(61^{\circ} 32^{\prime} \mathrm{N}, 14^{\circ} 13^{\prime} \mathrm{E}\right)$, alt ca $630 \mathrm{~m}$. Coll 1984 and subm by David Foster, Harvard Univ, Petersham, Massachusetts. Dated as part of proj for study of mire development in Dalarna and Västmanland in collaboration with Nils Malmer, Dept Plant Ecol, Univ Lund. Peat and gyttja samples taken with piston corer, diam $10 \mathrm{~cm}$.

\section{Lu-2423. Kräckelbäcken, Sample 1}

$$
\begin{array}{r}
\mathbf{1 6 4 0} \pm \mathbf{5 0} \\
\delta^{13} C=-25.0 \%
\end{array}
$$

Wood of pine root from stump exposed at base of peat along shore of broad pool. Sample depth ca lm. Comment: pretreated with HC1 and $\mathrm{NaOH}$. 
Lu-2424. Kräckelbäcken, Sample 2

$$
\begin{array}{r}
\mathbf{3 0 7 0} \pm \mathbf{5 0} \\
\delta^{13} C=-25.0 \%
\end{array}
$$

Wood from pine stump at base of peat, exposed along road cut. Stump rooted in mineral soil below $75 \mathrm{~cm}$ peat. Comment: pretreated with $\mathrm{HCl}$ and $\mathrm{NaOH}$.

\section{Lu-2425. Kräckelbäcken, Sample 3}

$$
\begin{array}{r}
\mathbf{2 9 2 0} \pm \mathbf{5 0} \\
\delta^{13} C=-24.9 \%
\end{array}
$$

Detrital gyttja, 30 to $40 \mathrm{~cm}$, above transition sedge peat (below $40 \mathrm{~cm}$ ) and gyttja $(0-40 \mathrm{~cm})$ in Fen Pool 1. Comment: pretreated with $\mathrm{HCl}$ and $\mathrm{NaOH}$.

\section{Lu-2426. Kräckelbäcken, Sample 4}

$$
\begin{array}{r}
\mathbf{3 5 1 0} \pm \mathbf{6 0} \\
\delta^{13} C=-25.5 \% \text { o }
\end{array}
$$

Sedge peat, 45 to $50 \mathrm{~cm}$, below transition peat/gyttja in Fen Pool 1 . Comment: pretreated with $\mathrm{HCl}$ and $\mathrm{NaOH}$. Charred in nitrogen atmosphere before burning.

\section{Lu-2427. Kräckelbäcken, Sample 5}

$$
\begin{array}{r}
\mathbf{3 4 1 0} \pm \mathbf{6 0} \\
\delta^{13} C=-26.5 \% 0
\end{array}
$$

Detrital gyttja, 50 to $55 \mathrm{~cm}$, above transition peat/gyttja in Fen Pool 2. Comment: pretreated with $\mathrm{HCl}$ and $\mathrm{NaOH}$. Charred in nitrogen atmosphere before burning.

Lu-2428. Kräckelbäcken, Sample 6

$$
\begin{array}{r}
1370 \pm 45 \\
\delta^{13} C=-26.0 \% 0
\end{array}
$$

Detrital gyttja, 15 to $20 \mathrm{~cm}$, above transition peat/gyttja in shallow part of Fen Pool 2. Comment: pretreated with HC1.

\section{Lu-2429. Kräckelbäcken, Sample 7}

$7830 \pm 70$

$\delta^{13} \mathrm{C}=-27.4 \%$ o HC1.

Basal sedge peat, 133 to $135 \mathrm{~cm}$, Core 2. Comment: pretreated with

\section{Lu-2430. Kräckelbäcken, Sample 8}

$$
\begin{array}{r}
\mathbf{6 2 8 0} \pm \mathbf{7 0} \\
\delta^{13} C=-27.8 \% 0
\end{array}
$$

Basal sedge peat, 113 to $115 \mathrm{~cm}$, Core 3. Comment: pretreated with $\mathrm{HC}$. Charred in nitrogen atmosphere before burning.

\section{Lu-2431. Kräckelbäcken, Sample 9} $\mathbf{5 8 8 0} \pm \mathbf{7 0}$

Basal sedge peat, 72 to $79 \mathrm{~cm}$, Core 4 . Comment: pretreated with $\mathrm{HCl}$ and $\mathrm{NaOH}$. Charred in nitrogen atmosphere before burning.

\section{Lu-2432. Kräckelbäcken, Sample 10}

$5410 \pm 60$ $\delta^{13} C=-27.3 \%$

Basal peat, 98 to $100 \mathrm{~cm}$, Core 5. Comment: pretreated with $\mathrm{HCl}$. Charred in nitrogen atmosphere before burning. 
Lu-2433. Kräckelbäcken, Sample 11

$5510 \pm 60$ Charred in nitrogen atmosphere before burning.

Lu-2434. Kräckelbäcken, Sample $12 \quad \delta^{13} C=-27.6 \% 0$
Basal peat, 78 to $80 \mathrm{~cm}$, Core 7 . Comment: pretreated with $\mathrm{HC} 1$.

$$
4670 \pm 60
$$
Charred in nitrogen atmosphere before burning.

\section{Lu-2435. Kräckelbäcken, Sample 13}

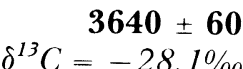

Basal peat, 83 to $89 \mathrm{~cm}$, Core 8 . Comment: pretreated with $\mathrm{HCl}$ and $\mathrm{NaOH}$. Charred in nitrogen atmosphere before burning. Sample undersized; diluted; $82 \%$ sample.

\section{Lu-2436. Kräckelbäcken, Sample 14}

$$
\begin{array}{r}
6980 \pm 70 \\
\delta^{13} C=-27.8 \%
\end{array}
$$

Basal peat, 94 to $100 \mathrm{~cm}$, Core 9 . Comment: pretreated with $\mathrm{HCl}$ and $\mathrm{NaOH}$. Charred in nitrogen atmosphere before burning.

\section{Lu-2437. Kräckelbäcken, Sample 15}

$$
4680 \pm 60
$$
Core 10. Commen: preen Core 10. Comment: pretreated with $\mathrm{HCl}$ and $\mathrm{NaOH}$. Charred in nitrogen atmosphere before burning.

\section{Lu-2438. Kräckelbäcken, Sample 16}

$\mathbf{9 0 4 0} \pm \mathbf{8 0}$

Basal peat, 424 to $430 \mathrm{~cm}$, Core 11. Comment: pretreated with $\mathrm{HC} 1$ and $\mathrm{NaOH}$. Charred in nitrogen atmosphere before burning.

\section{Lu-2439. Kräckelbäcken, Sample 17}

$\mathbf{7 8 4 0} \pm \mathbf{8 0}$

Basal peat, 114 to $120 \mathrm{~cm}$, Core 12. Comment: pretreated with $\mathrm{HC} 1$ and $\mathrm{NaOH}$. Charred in nitrogen atmosphere before burning.

\section{Lu-2463. Odensjön, Sample 1, BP 2}

$$
14,800 \pm 180
$$

$\delta^{13} C=-43.5 \%$

Water mosses separated from clay gyttja, 514 to $520 \mathrm{~cm}$ below sediment surface in Odensjön (Rapp, 1984, p 21$)$, Scania $\left(56^{\circ} 00^{\prime} 15^{\prime \prime} \mathrm{N}, 13^{\circ} 16^{\prime}\right.$ $45^{\prime \prime}$ E). Coll 1984 by B Berglund; subm by B Berglund and T Persson, Dept Quaternary Geol, Univ Lund. Sample taken with piston core sampler, diam $7 \mathrm{~cm}$. Water depth at sampling point $18.7 \mathrm{~m}$. Dated to confirm suspected hardwater or old groundwater effect shown by dated surface sediment and recent submersed macrophytes (Håkansson, 1977, p 438-440, 1979). Sample from upper part of Aller $\phi \mathrm{d}$ pollen zone. No pretreatment; sample 
undersized; diluted; $79 \%$ sample. Comment: date $>3000$ yr too early because of suspected effects.

\section{Lu-2466. Nässja I, 1984}

$3440 \pm 60$

Muddy, highly humified fen peat, 100 to $105 \mathrm{~cm}$ below peat surface at Nässja, Östergötland $\left(58^{\circ} 20^{\prime} \mathrm{N}, 14^{\circ} 50^{\prime} \mathrm{E}\right)$. Coll 1984 by $\mathrm{H}$ Göransson and G Lemdahl; subm by H Göransson, Dept Quaternary Geol, Univ Lund. Dated as complement to Dags Mosse series (R, 1983, v 25, p 877-880; 1984, v 26, 393-394). Comment: pretreated with HC1.

\section{Lu-2470. Krageholmssjön}

$6130 \pm 70$

Clay gyttja from $690 \mathrm{~cm}$ below sediment surface in Krageholmssjön, $10 \mathrm{~km}$ NNW of Ystad, Scania $\left(55^{\circ} 30^{\prime} \mathrm{N}, 13^{\circ} 45^{\prime} \mathrm{E}\right)$. Coll 1985 and subm by J Regnell, Dept Quaternary Geol, Univ Lund. Sample pooled from same level in 2 cores taken with Livingstone sampler, diam $6 \mathrm{~cm}$. Water depth $7.35 \mathrm{~m}$ at sampling point. Sample just below Ulmus decline according to pollen analysis. Dated to look for possible hardwater effect. Pretreated with HC1. Comment: hardwater error ca $1000 \mathrm{yr}$.

\section{Vasasjön series (II)}

Peat and gyttja from ancient Vasasjön, ca $4 \mathrm{~km} \mathrm{~N}$ of Sövestad, S Scania $\left(55^{\circ} 32.2^{\prime} \mathrm{N}, 13^{\circ} 48.3^{\prime} \mathrm{E}\right)$. Coll 1982 and subm by M Hjelmroos, Dept Quaternary Geol, Univ Lund. Dated as complement to Vasasjön series (R, 1986, v 28, p 145). Depths given are below present surface. All samples pretreated with HCil and charred in nitrogen atmosphere before burning.

Lu-2471. Vasasjön 1:85, 294 to $296 \mathrm{~cm}$ $3770 \pm 60$

Slightly humified peat.

Lu-2472. Vasasjön 2:85, 353 to $356 \mathrm{~cm}$ $\delta^{13} C=-28.5 \%$

Slightly humified peat.

Lu-2473. Vasasjön 3:85, 368 to $371 \mathrm{~cm}$

$$
\begin{array}{r}
\mathbf{3 8 4 0} \pm \mathbf{6 0} \\
\delta^{13} C=-29.2 \% 0
\end{array}
$$

Moderately humified peat. Late part of Early Subboreal time according to pollen analysis.

Lu-2474. Vasasjön 4:85, 389 to $391 \mathrm{~cm}$

$$
\begin{array}{r}
\mathbf{4 3 1 0} \pm \mathbf{6 0} \\
\delta^{13} C=-30.7 \% 0
\end{array}
$$

Moderately humified peat. Middle part of Subboreal time.

Lu-2475. Vasasjön 5:85, 407 to $410 \mathrm{~cm}$ $4360 \pm 60$

Moderately to highly humified peat. Middle part of Early Subboreal time. 


\section{Lu-2476. Vasasjön 6:85, 432 to $435 \mathrm{~cm}$}

$4630 \pm 60$

Highly humified peat. First part of Early Subboreal time.

Lu-2477. Vasasjön 7:85, 509 to $510 \mathrm{~cm}$

Gyttja. Late part of Atlantic time.

\section{Lu-2383. Bysjön}

Peat from 8.1 to $8.2 \mathrm{~m}$ below sediment surface in core from Lake Bysjön, 25km E of Lund, Scania (55 $\left.41^{\prime} \mathrm{N}, 13^{\circ} 33^{\prime} \mathrm{E}\right)$. Coll 1983 and subm by $G$ Digerfeldt, Dept Quaternary Geol, Univ Lund. Peat is overlain by ca $8 \mathrm{~m}$ lake sediments. Water depth $4.85 \mathrm{~m}$ at coring point. Comment: pretreated with $\mathrm{HCl}$ and $\mathrm{NaOH}$.

\section{Bjäresjö series}

Peat and gyttja from lake S of Bjäresjö village, $5 \mathrm{~km} \mathrm{NW}$ of Ystad, S Scania $\left(55^{\circ} 27.5^{\prime} \mathrm{N}, 13^{\circ} 45.3^{\prime} \mathrm{E}\right)$. Coll 1985 and subm by M-J Gaillard, Dept Quaternary Geol, Univ Lund. Dated as part of study of local vegetational changes during last $6000 \mathrm{yr}$ in Ystad area. For previous date from lake, see R, 1983, v 25, p 877. Samples are from Core No. 3, taken with Russian-type corer, diam $10 \mathrm{~cm}$. Depts $(\mathrm{cm})$ refer to sediment surface. Water depth ca $1.7 \mathrm{~m}$ at coring point. All samples pretreated with HC1.

\section{Lu-2486. Bjäresjö 1:85, 369 to $375 \mathrm{~cm}$}

Moderately humified moss peat.

\section{Lu-2487. Bjäresjö 2:85, 361 to $364 \mathrm{~cm}$}

Moderately humified moss peat with remains of Cyperaceae (?). Comment: sample undersized; diluted; $77 \%$ sample.

Lu-2488. Bjäresjö 3:85, 357 to $360 \mathrm{~cm}$

$\mathbf{2 7 6 0} \pm \mathbf{5 0}$

$\delta^{13} C=-25.8 \%$

Coarse detritus gyttja with many Potamogeton fruits and remains of Cyperaceae.

Lu-2489. Bjäresjö 4:85, 302 to $305 \mathrm{~cm}$

$$
\delta^{13} \mathrm{C}=-29.4 \% \text { o }
$$

Silty drift gyttja with some sand. Pollen zone SA 1 (Nilsson, 1964).

\section{Lu-2490. Bjäresjö 5:85, 270 to $273 \mathrm{~cm}$}

$1310 \pm 45$

$\delta^{13} \mathrm{C}=-29.5 \%$

Drift gyttja (coarse detritus) with fruits of Potamogeton and rootlets. Pollen zone boundary SA 1/SA 2. 
Lu-2491. Bjäresjö 6:85, 264.5 to $267.5 \mathrm{~cm}$

$1240 \pm 45$

$\delta^{13} C=-29.3 \% 0$

Clayey drift gyttja (coarse detritus) with fruits of Potamogeton. Pollen zone SA 2.

\section{Iceland}

\section{Icelandic Subfossil Marine Shell Series IV}

Marine bivalve, gastropod, and balanid shells from W Iceland. Coll 1980 and 1983 and subm by Ó Ingólfsson, Dept Quaternary Geol, Univ Lund. Dated as complement to previous series from area (R, 1983, v 25, p $882 ; 1984$, v 26, p 398-399; 1986, v 28, p 152). Preliminary rept pub by Ingólfsson (1985).

\section{Lu-2371. Árdalsá 2}

$12,510 \pm 140$

Shell fragments (Balanus sp, Nucula tenuis, Macoma calcarea, Hiatella arctica, Mya truncata) from massive marine silt sequence with sand laminae and occasional dropstones at Árdalsá R, lower part of Borgarfjördur $\left(64^{\circ}\right.$ $\left.32^{\prime} \mathrm{N}, 21^{\circ} 48^{\prime} \mathrm{W}\right)$. Mollusks probably in situ at ca $+47 \mathrm{~m}$. Comment: outer $14 \%$ removed by acid leaching. Sample undersized; diluted; $71 \%$ sample.

\section{Lu-2372. Āsbákkar-Ásgil 2}

$12,080 \pm 120$

Shells (Astarte elliptica) and shell fragments (Hiatella arctica, Mya truncata, Chlamys islandica) from glacial-marine silt between 2 till beds; $\mathrm{ca}+3$ to $4 \mathrm{~m}$ at Ásbakkar, $\mathrm{N}$ of Akranes $\left(64^{\circ} 24^{\prime} \mathrm{N}, 22^{\circ} 02^{\prime} \mathrm{W}\right)$. Shells not in situ. Sample from same stratigraphic position dated at 11,980 $\pm 130 \mathrm{BP}$ (Lu2196 ; R, 1984, v 26, p 399). Comment: outer $13 \%$ removed by acid leaching. Sample undersized; diluted; $85 \%$ sample.

\section{Lu-2373. Ásbakkar-Āsgil 3}

$11,910 \pm 140$

Shell fragments (Chlamys islandica, Buccinum sp, Trophon sp, Balanus sp) from same site and same strat position as Lu-2372, above. Comment: outer $10 \%$ removed by acid leaching. Sample undersized; diluted; $67 \%$ sample.

\section{Lu-2374. Skipanes 2}

$12,250 \pm 100$

$\delta^{13} \mathrm{C}=+1.2 \%$

Shells (Hiatella arctica, Astarte elliptica, Mya truncata, Chlamys islandica, Macoma calcarea, Balanus sp) from glacial-marine silt below ice-contact complex; +3 to $4 \mathrm{~m}$ at Skipanes, Melasveit, $\mathrm{N}$ of Akranes $\left(64^{\circ} 24^{\prime} \mathrm{N}, 21^{\circ} 54^{\prime} \mathrm{W}\right)$. Shells probably in situ. Sample from top of same sedimentary sequence dated at 10,370 \pm 90 BP (Lu-2197; R, 1984, v 26, p 399). Comment: outer $27 \%$ removed by acid leaching. 


\section{Lu-2378. Skipanes 3}

$10,520 \pm 150$

Shells (Macoma calcarea, Mya truncata, Tectonatica affinis) from marine silt; +3 to $4 \mathrm{~m}$ at Skipanes, $\mathrm{N}$ of Akranes $\left(64^{\circ} 24^{\prime} \mathrm{N}, 21^{\circ} 54^{\prime} \mathrm{W}\right)$. Shells in situ in silt deposited after retreat of glaciers from area. Sample from intermediate strat position between Lu-2197 and -2374, above. Comment: outer $17 \%$ removed by acid leaching. Sample undersized; diluted; $53 \%$ sample.

\section{Lu-2375. Melabakkar-Melar 1}

$12,350 \pm 120$

Shell fragments (Chlamys islandica, Mya truncata, Balanus balanus, and unid. sp) from marine silt, +3 to $20 \mathrm{~m}$, underlain by till and overlain by littoral sand at Melabakkar, below Melar farm, Melasveit, $\mathrm{N}$ of Akranes $\left(64^{\circ} 25^{\prime}\right.$ $\left.\mathrm{N}, 22^{\circ} 02^{\prime} \mathrm{W}\right)$. Shells not in situ. Comment: outer $10 \%$ removed by acid leaching. Sample undersized; diluted; $88 \%$ sample.

\section{Lu-2376. Ásbakkar 2}

$11,830 \pm 100$

$\delta^{13} \mathrm{C}=+0.8 \%$ o

Shells (Mya truncata, Hiatella arctica) from glacial-marine silt, ca $+7 \mathrm{~m}$, overlain by till complex at Ásbakkar, $\mathrm{N}$ of Akranes $\left(64^{\circ} 24^{\prime} \mathrm{N}, 22^{\circ} 02^{\prime} \mathrm{W}\right)$. Shells probably in situ. Comment: outer $17 \%$ removed by acid leaching.

\section{Lu-2377. Ásbakkar 3}

$12,310 \pm 110$

$\delta^{13} C=+0.6 \%$

Shells (Chlamys islandica, Balanus balanus) and shell fragments (Hiatella arctica, Astarte montagui, Trophon sp, Balanus sp) from glacial-marine silt in complex deposits of ice-contact debris; +3 to $4 \mathrm{~m}$ at Ásbakkar, near Sulunes, $\mathrm{N}$ of Akranes $\left(64^{\circ} 23^{\prime} \mathrm{N}, 2^{\circ} 01^{\prime} \mathrm{W}\right)$. Shells probably not in situ. Comment: outer $40 \%$ removed by acid leaching.

\section{Lu-2379. Ás}

$12,380 \pm 110$

$\delta^{13} \mathrm{C}=+1.3 \%$

Shells (Chlamys islandica) from glacial-marine silt below complex till sequence, ca $+25 \mathrm{~m}$ at Ás in Melasveit, $\mathrm{N}$ of Akranes $\left(64^{\circ} 24^{\prime} \mathrm{N}, 22^{\circ} 02^{\prime} \mathrm{W}\right)$. Shells probably in situ. Comment: outer $54 \%$ removed by acid leaching. General Comment: corrections for deviations from $\delta^{13} \mathrm{C}=-25 \%$ PDB are applied. No corrections are made for reservoir age of living marine mollusks. Reservoir age for coastal waters of Iccland pub by Håkansson (1983b).

\section{Akranes series}

Sandy peat from 0.7 to $3.2 \mathrm{~m}$ below present high-tide marks at Höfdavik, just $\mathrm{N}$ of Akranes $\left(64^{\circ} 20^{\prime} \mathrm{N}, 22^{\circ} 04^{\prime} \mathrm{W}\right)$. Coll 1983 and subm by () Ingólfsson. Samples pretreated with $\mathrm{HCl}$ and $\mathrm{NaOH}$.

\section{Lu-2395. Akranes 1}

$6130 \pm 80$

Sandy peat, 295 to $320 \mathrm{~cm}$ below present high-tide marks. Comment: sample undersized; diluted; $75 \%$ sample. 
Lu-2396. Akranes 2

Sandy peat, 70 to $95 \mathrm{~cm}$ below present high-tide marks. Comment: sample undersized; diluted; $39 \%$ sample.

\section{Southern Iceland Series I}

Marine bivalve and balanid shells from S Iceland, coll 1982-1983 and subm by A Hjartarsson, Natl Energy Authority, Reykjavik, Iceland. Dated as part of study of Late Weichselian glacial stratigraphy and chronology of low alt areas around R Thjórsá, S Iccland.

\section{Lu-2399. Sogíđ, Bíldsfell}

$$
9420 \pm 80
$$

Two articulated shell pairs (Cyprina islandica) from stratified silt outside end moraines of “Grafningur"stage, + ca $20 \mathrm{~m}$ at Sogíd $\left(64^{\circ} 03^{\prime} \mathrm{N}, 20^{\circ}\right.$ $59^{\prime} \mathrm{W}$ ), near Bíldsfell. Comment: outer $30 \%$ removed by acid leaching.

\section{Lu-2400. Dynjandi in Brùará}

$$
\begin{array}{r}
\mathbf{1 0 , 1 9 0} \pm \mathbf{9 0} \\
\delta^{13} C=+0.9 \%
\end{array}
$$

Shells and large fragments (Mya truncata) from stratified silt inside end moraines of "Buđi" stage (Kjartansson, Thorarinsson \& Einarsson, 1964; Norddahl, 1983, p 6), + ca 60m at Dynjandi in Brùará $\left(64^{\circ} 09^{\prime} \mathrm{N}, 20^{\circ} 34^{\prime}\right.$ W). Comment: outer $51 \%$ removed by acid leaching.

\section{Lu-2401. Stóra Laxá at Hrepphólar 1}

$$
10,110 \pm 140
$$

Permula) in situ in stratificd silt underlain by till of "Hólakot" stage (Kjartansson, 1943), +65 to $70 \mathrm{~m}$ at Hrepphólar, Stóra Laxá $\left(64^{\circ} 03^{\prime} \mathrm{N}, 20^{\circ} 20^{\prime} \mathrm{W}\right)$. Comment: outer $10 \%$ removed by acid leaching. Sample undersized; diluted; $52 \%$ sample.

\section{Lu-2402. Stóra Laxá at Hrepphólar 2}

$$
9960 \pm 160
$$

Shell fragments (Balanus sp) from till of "Hólakot" stage, + ca $65 \mathrm{~m}$ at Hrepphólar, Stóra Laxá $\left(64^{\circ} 03^{\prime} \mathrm{N}, 20^{\circ} 20^{\prime} \mathrm{W}\right)$. Coll May 1982, Sept and Oct 1983. Comment: outer 9\% removed by acid leaching. Sample undersized; diluted; $44 \%$ sample.

\section{Lu-2403. Thjórsá 1}

$$
10,360 \pm 90
$$

$\delta^{13} \mathrm{C}=+0.8 \%$

Shells (Mya truncata, Balanus sp) from stratified sand bed overlain by till of "Buđi" stage (Younger Dryas), + ca $60 \mathrm{~m}$ between Thràndarholt and Miđhús farms $\left(64^{\circ} 02^{\prime} \mathrm{N}, 20^{\circ} 20^{\prime} \mathrm{W}\right)$ (Áskelsson, 1934). Comment: outer $44 \%$ removed by acid leaching.

\section{Lu-2404. Thjórsá 2, near Minnahof}

$\mathbf{1 0 , 2 2 0} \pm \mathbf{9 0}$

Shells (Balanus sp) found in situ on tillite overlain by till of "Buđi" 
stage (Younger Dryas), + ca 65m near Minnahof bei Thjórsá R $\left(64^{\circ} 0 \mathrm{I}^{\prime} \mathrm{N}\right.$, $\left.20^{\circ} 18^{\prime} \mathrm{W}\right)$.

\section{Lu-2405. Rauđalækur, Rangarthing}

$10,190 \pm 130$

Shells and fragments (Chlamys islandica) from sandy layer in raised delta of Rángárvellir (Kjartansson, 1943) at Rauđalækur, Rangarthing $\left(63^{\circ}\right.$ $\left.50^{\prime} \mathrm{N}, 20^{\circ} 28^{\prime} \mathrm{W}\right)$. Comment: outer $10 \%$ removed by acid leaching. Sample undersized; diluted; $62 \%$ sample.

\section{Lu-2406. Ytri-Rangá at Bjarg}

$10,380 \pm 90$

Shell fragments (Hiatella arctica, Balames sp) from sand layer in raloc delta of Rángárvellir, +30 to $35 \mathrm{~m}$ at Bjarg, Ytri-Rangá $\left(63^{\circ} 50^{\prime} \mathrm{N}, 20^{\circ} 25^{\prime}\right.$ W). Comment: outer $33 \%$ removed by acid leaching.

General Comment: corrections for deviations from $\delta^{13} \mathrm{C}=-25 \%$ PDB are applied. No corrections are made for reservoir age of living marine mollusks. Reservoir age for coastal waters of Iceland pub by Håkansson (1983b).

\section{Spitsbergen}

\section{Bohemanflya series (II)}

Marine bivalve shells from Bohemanflya, Isfjorden, W Spitsbergen. Coll 1984 by members of Univ Lund field expedition to Spitsbergen; subm by C Hjort, Dept Quaternary Geol, Univ Lund. For other dates from area, see R, 1984, v 26, p 399-400.

\section{Lu-2363. Bohemanflya 5}

$$
\begin{array}{r}
9650 \pm 90 \\
\delta^{13} C=+1.2^{\circ} \% 0
\end{array}
$$

Shells (Mya truncata) from solifluction deposit in recent coastal cliff at Bohemanflya $\left(78^{\circ} 26^{\prime} \mathrm{N}, 14^{\circ} 40^{\prime} \mathrm{E}\right)$; + 0 to $\mathrm{lm}$. Comment: outer $60 \%$ removed by acid leaching.

\section{Lu-2364. Bohemanflya 6}

$$
\begin{array}{r}
9950 \pm \mathbf{9 0} \\
\delta^{13} C=+0.7 \% 0
\end{array}
$$

Shells (Hiatella arctica) from frostballs on " $20 \mathrm{~m}$-plane." Previous date on shell from alt 18 to $20 \mathrm{~m}$ is $9630 \pm 90 \mathrm{BP}(\mathrm{Lu}-2138$; R, 1984, v 26, p 399). Comment: outer $64 \%$ removed by acid leaching.

General Comment: corrections for deviations from $\delta^{13} \mathrm{C}=-25 \%$ PDB are applied. No corrections are made for reservoir age of living marine mollusks. Revised reservoir age for coastal waters of Spitsbergen pub by Olsson (1980, fig 6, p 673).

\section{German Federal Republic}

\section{River Schlei series}

Sediment from estuary of R Schlei, near town of Schleswig, Schleswig-

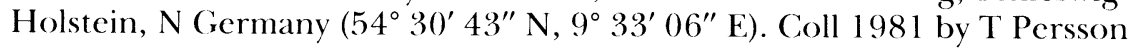


and M Enell; subm by W Ripl, Dept Limnol, Technol Univ, Berlin. Dated to establish chronology of sediment used for study of environmental changes in area. Coring point in comparatively sheltered position. Water depth $1.5 \mathrm{~m}$. Samples taken with Livingstone sampler, diam $6 \mathrm{~cm}$. Depths $(\mathrm{cm})$ refer to sediment surface. Samples pretreated with $\mathrm{HCl}$; undersized; diluted.

\section{Lu-2407. R Schlei Estuary 1}

$4990 \pm 70$

Gyttja, 405 to $412.5 \mathrm{~cm}$, Sample AP $76-77$ and AP 77. Comment: $76 \%$ sample.

$$
\delta^{13} \mathrm{C}=-29.5 \%
$$

Lu-2446. R Schlei Estuary 2

$4650 \pm 80$

$\delta^{13} \mathrm{C}=-28.0 \%$ sample.

Gyttja, 305 to $312.5 \mathrm{~cm}$, Sample AP 56-57 and AP 57. Comment: $58 \%$

Lu-2447. R Schlei Estuary 3

$\mathbf{3 5 6 0} \pm \mathbf{8 0}$
$\delta^{13} C=-27.6 \% 0$ sample.

Gyttja, 235 to $242.5 \mathrm{~cm}$, Sample AP $42-43$ and AP 43. Comment: $58 \%$

\section{Lu-2408. R Schlei Estuary 4}

$$
\begin{array}{r}
\mathbf{2 3 5 0} \pm \mathbf{5 0} \\
\delta^{13} C=-29.5 \% 0
\end{array}
$$

Gyttja, 145 to $152.5 \mathrm{~cm}$, Sample AP 24-25 and AP 25. Comment: $91 \%$ sample.

\section{Bulgaria}

\section{Lake Blatniza Series II}

Sediment and peat from Lagoon-lake Blatniza (Durankulak), W shore

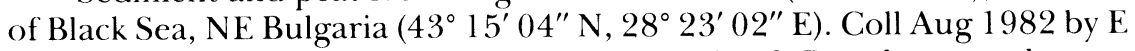
Bozilova, Biol Fac, Univ Sofia; subm by B Berglund. Dated as complement to Lake Blatniza Series I (R, 1986, v 28, p 153-154). Depths refer to sediment surface. Samples pretreated with $\mathrm{HC} 1$.

\section{Lu-2381. Lake Blatniza, 400 to $420 \mathrm{~cm}$}

$6170 \pm 150$

Calcareous gyttja with small organic content. NAP pollen (Artemisia, Chenopodiaceae, Poaceae) dominating. Sample pooled from 2 core pieces (diam ca $2.5 \mathrm{~cm}$ ) from same level. Comment: prolonged HC1 treatment necessary for removal of carbonates. Sample undersized; diluted; $33 \%$ sample.

Lu-2382. Lake Blatniza, 200 to $215 \mathrm{~cm}$

$2290 \pm 50$

Phragmites peat. Pollen of Quercetum mixtum, Chenopodiaceae, Poaceae, and Asteraceae indicating Subatlantic time. 
Czechoslovakia

\section{Zlatnická dolina series}

Samples from peat bog ca $5 \mathrm{~km}$ NW of village of Mútné, near Dolný Kubin (district town), NE Czechoslovakia $\left(49^{\circ} 31^{\prime} \mathrm{N}, 19^{\circ} 17^{\prime} \mathrm{E}\right.$ ), alt ca $860 \mathrm{~m}$. Coll 1977 by E Rybníčková and K Rybníček; subm by E Rybníčková, Bot Inst, Czechoslovak Acad Sci, Brno. For other dates from area, see Bobrov series (R, 1982, v 24, p 200-201; 1986, v 28, p 153). All samples pretreated with HC1. Depths refer to present bog surface. Core designation OK-9-A. Pollen zones after Firbas (1949).

Lu-2410. Zlatnicka dolina, 330 to $335 \mathrm{~cm}$

$7450 \pm 130$

Moderately humified clayey peat with small wood fragments. Older than Atlantic period (9000-7000 BP). Comment: sample undersized; diluted; $46 \%$ sample.

Lu-2411. Zlatnicka dolina, 299 to $303 \mathrm{~cm}$

$6060 \pm 70$

Highly humified peat with small clay content. Pollen zone AT 2.

Lu-2412. Zlatnicka dolina, 270 to $272 \mathrm{~cm}$

$4300 \pm 70$

Moderately humified Carex peat. Beginning curve Subboreal pollen sample.

\section{Lu-2413. Zlatnicka dolina, 248 to $250 \mathrm{~cm}$}

$$
3800 \pm 60
$$

Moderately humified Carex peat with Phragmit bound boundary of Older Subatlantic pollen zone. First traces of human activity in area. Comment: $>1000$ yr older than expected.

\section{Lu-2414. Zlatnicka dolina, 48 to $50 \mathrm{~cm}$}

$1030 \pm 45$

Older Suphom peat with many rooter and Older Subatlantic pollen zones. Beginning of closed pollen curve of Cerealia; decrease of Abies pollen percentages.

\section{Jamaica}

\section{Black River Morass Series III}

Peat from coastal wetland at Black R, S Jamaica $\left(18^{\circ} 05^{\prime} \mathrm{N}, 77^{\circ} 50^{\prime} \mathrm{W}\right)$. Coll 1982 and subm by G Digerfeldt, Dept Quaternary Geol, Univ Lund. Dated as complement to Black River Morass series (R, 1982, v 24, p 203; 1984, v 26, p 401-404). Depths given are below present surface. Pretreated with $\mathrm{HC} 1$. 
Lu-2440. Black R Morass, B 14, 55 to $65 \mathrm{~cm}$

$$
\triangle_{(\mathrm{NBS} \mathrm{1950)}}=-\mathbf{8 . 0} \pm \mathbf{5 . 1} \%
$$

Moderately humified sedge peat. Comment: modern sample. For definition of $\triangle_{(\text {NBS 1950) }}$, see R, 1981, v 23, p 394.

Lu-2441. Black R Morass, B 3, 140 to $150 \mathrm{~cm}$

$3850 \pm 60$

Moderately humified sedge peat.

\section{ARCHAEOLOGIC SAMPLES}

Sweden

$$
\delta^{13} C=-25.8 \%
$$

\section{Lu-2384. Kroknäs, SM 6250}

$$
\begin{array}{r}
\mathbf{4 2 0 0} \pm \mathbf{6 0} \\
\delta^{13} C=-25.8 \%
\end{array}
$$

Wood (Pinus sp) id by T Bartholin, from paddle from Kroknäs, Skellefteå, N Sweden $\left(64^{\circ} 32^{\prime} \mathrm{N}, 21^{\circ} 12^{\prime} \mathrm{E}\right)$. Coll before 1945 by workers during ditching; subm by $\mathrm{P}$ Gustafsson, Skellefteå Mus, Skellefteå. Paddle seems to be worked by stone tools and old notes about pollen analysis indicated "transition Stone/Bronze Age." Comment: pretreated with HCl and $\mathrm{NaOH}$.

\section{Kämpinge series}

Charcoal from excavation of Medieval settlement area probably connected with adjacent earthwork (wall) built of turf and stone in 2 stages at Kämpinge village, $5 \mathrm{~km} \mathrm{~S}$ of Foteviken, SW Scania $\left(55^{\circ} 24^{\prime} \mathrm{N}, 12^{\circ} 59^{\prime} \mathrm{E}\right)$. Coll 1983 and subm by L Ersgård, Inst Archaeol, Univ Lund. Study is continuation of survey work 1982 at Foteviken (R, 1983, v 25, p 888; 1984, v 26, p 408-409). All samples pretreated with HCl1 and $\mathrm{NaOH}$.

\section{Lu-2385. Kämpinge 1:1983}

$1000 \pm 45$

Charcoal from cultural layer, $\operatorname{Tr} \mathrm{C}$.

$$
\delta^{13} \mathrm{C}=-26.0 \%
$$

Lu-2386. Kämpinge 2:1983

$900 \pm 45$

Charcoal from hearth, $\operatorname{Tr}$ C.

$$
\delta^{13} C=-26.2 \%
$$

Lu-2387. Kämpinge 3:1983

$$
850 \pm 45
$$

Sample from charcoal concentration at basal part of cultural layer, $\operatorname{Tr}$ C.

Lu-2388. Kämpinge Sandeplan 4:1983

$$
\begin{array}{r}
\mathbf{2 9 7 0} \pm \mathbf{5 0} \\
\delta^{13} C=-25.8 \% 0
\end{array}
$$

Charcoal from cultural layer at Kämpinge Sandeplan $\left(55^{\circ} 25^{\prime} \mathrm{N}, 12^{\circ}\right.$ $\left.59^{\prime} \mathrm{E}\right)$. Assoc with artifact assemblage indicating Bronze Age. 


\section{Skateholm Series V}

Charcoal from settlement area and grave field (Early Erteb $\varnothing l l e$ culture) at Skateholm, Tullstorp parish, S Scania $\left(55^{\circ} 23^{\prime} \mathrm{N}, 13^{\circ} 29^{\prime} \mathrm{E}\right)$. Coll $1983-$ 1984; subm by L Larsson, Inst Archaeol, Univ Lund. Dated as complement to previous Skateholm series (R, 1982, v 24, p 205-206; 1983, v 25, p 887; 1984, v 26, p 405-406; 1986, v 28, p 155).

\section{Lu-2443. Skateholm II, Grave IX}

$$
5460 \pm 110
$$

Charcoal assoc with Construction 17, Grave IX (Larsson, Coll 1983 by R Ristmar. Comment: no pretreatment; diluted; $41 \%$ sample.

\section{Lu-2444. Skateholm II, Grave XV}

$5900 \pm 140$

Charcoal from Construction 33, Grave XV (Larsson, 1984, p 62-64). Coll 1984 by A Götz. Comment: no pretreatment; sample undersized; diluted; $21 \%$ sample. (4 1-day counts.)

\section{Lu-2445. Skateholm II, Construction 16}

$5660 \pm 110$

Charcoal from Construction 16 , above grave. Coll 1983 Comment: no pretreatment; sample undersized; diluted; $33 \%$ bam A Göz. day counts.)

\section{Lu-2478. Skateholm II, Construction III}

$$
6300 \pm 100
$$

Charcoal from Construction III. Coll 1984 by A Carlie. Comment: mild pretreatment with $\mathrm{NaOH}$ and $\mathrm{HC} 1$. Sample undersized; diluted; $43 \%$ sample. (3 1-day counts.)

\section{Bredasten series}

Charcoal from settlement area (Erteb $\phi$ lle culture) at Bredasten, E part of Öja-Herrestad mosse, ENE of Ystad (55 $\left.27^{\prime} \mathrm{N}, 13^{\circ} 55.5^{\prime} \mathrm{E}\right)$. Coll 1984 by M Larsson and A Carlie; subm by L Larsson. Assoc artifact assemblage indicates about same age as for earliest use of Skateholm I (Larsson, 1984, p 76). Samples received mild pretreatment with $\mathrm{NaOH}$ and $\mathrm{HCl}$.

\section{Lu-2420. Bredasten, Construction 14}

$$
\mathbf{5 3 4 0} \pm \mathbf{7 0}
$$

Charcoal (Fraxinus, Prunus, Corylus, Alnus, \& Pomoideae) id by T Bartholin, from Construction 14, lower layer. Comment: sample undersized; diluted; $77 \%$ sample.

\section{Lu-2422. Bredasten, Construction 4}

$$
\begin{array}{r}
\mathbf{5 1 7 0} \pm \mathbf{6 0} \\
\delta^{13} C=-27.2 \% 0
\end{array}
$$

Charcoal (Corylus, Alnus, Pinus, Fraxinus, Prunus, \& Pomoideae) id by T Bartholin, from Sq F, Construction 4 ("Ringen"). 
General Comment (LL): dates younger than expected judging from assoc archaeol finds.

\section{Östra Vemmenhög series (II)}

Charcoal from settlement area at Ö Vemmenhög 7:40, S Scania $\left(55^{\circ}\right.$ $23^{\prime} 30^{\prime \prime} \mathrm{N}, 13^{\circ} 29^{\prime} 20^{\prime \prime} \mathrm{E}$ ). Coll 1984 and subm by L Larsson. Samples are from cultural layer with artifact assemblage indicating Funnel Beaker culture, Middle Neolithic Period IV. For other dates from Ö Vemmenhög, see $\mathrm{R}, 1986$, v 28, p 155-156. Pretreated with $\mathrm{HCl}$ and $\mathrm{NaOH}$.

Lu-2397. Ö Vemmenhög 7:40/1

$4130 \pm 70$

Charcoal from Sq $\mathrm{x}=104, \mathrm{y}=119$. Assoc with pottery and flint artifacts.

\section{Lu-2398. Ö Vemmenhög 7:40/2}

$3470 \pm 60$

$\delta^{13} \mathrm{C}=-26.4 \%$

Charcoal from Sqs $\mathrm{x}=104, \mathrm{y}=117 ; \mathrm{x}=104, \mathrm{y}=118$. Assoc with pottery and flint artifacts. Comment (LL): younger than expected.

\section{Lu-2464. St Beddinge, No. 27476:N}

$3740 \pm 60$
$\delta^{13} C=-21.1 \%$

Collagen from human bone from grave at St Beddinge, S Scania $\left(55^{\circ}\right.$ $23^{\prime} \mathrm{N}, 13^{\circ} 27^{\prime} \mathrm{E}$ ). Coll 1936 by F Hansen; subm by L Larsson. Grave finds indicate Battle Axe culture (Malmer, M P, 1962). Comment: organic carbon content: $1 \%$. Collagen extracted as described previously (R, 1976, v 18, p 290) without $\mathrm{NaOH}$ treatment. Sample undersized; diluted; $91 \%$ sample.

\section{St Köpinge Series II}

Charcoal from Bronze and Iron Age sites in St Köpinge parish, S Scania. Dated as complement to St Köpinge Series I (R, 1986, v 28, 156158). Coll 1979 and 1982 (Lu-2449) and subm by S Tesch, Inst Archaeol, Univ Lund. Charcoal id by T Bartholin, Dept Quaternary Geol, Univ Lund.

\section{Lu-2451. Köpinge 15:22, A 333}

$1250 \pm 45$
$\delta^{13} C=-27.5 \% 0$

Charcoal (Betula sp) from hearth at Köpinge 15:22 (55 $26^{\prime} \mathrm{N}, 13^{\circ} 59^{\prime}$ E). Assoc artifacts indicate Iron Age. Comment: pretreated with $\mathrm{HC} 1$ and $\mathrm{NaOH}$.

Lu-2450. Köpinge 15:22, A 435

$$
\begin{array}{r}
1140 \pm \mathbf{4 5} \\
\delta^{13} C=-27.2 \% 0
\end{array}
$$

Charcoal (Alnus sp) from hearth at same site as Lu-2451, above. Assoc artifacts indicate Iron Age. Comment: pretreated with $\mathrm{HCl}$ and $\mathrm{NaOH}$.

Lu-2460. L:a Köpinge 19:1, A 30

$2530 \pm 90$

Charcoal (Quercus sp, Fraxinus sp, \& Pinus sp) from Bronze Age pit at 


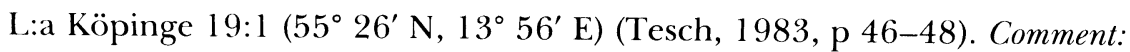
no pretreatment; sample undersized; diluted; $42 \%$ sample.

\section{Lu-2461. L:a Köpinge 19:1, A 216}

$2790 \pm 60$

Charcoal (Quercus sp, Corylus avellana, Alnus sp, Fraxinus excelsior, \& Betula sp) from hearth in House II at same site as Lu-2460, above. Assoc artifacts indicate Late Bronze or Early Iron Age. Comment: pretreated with $\mathrm{HCl}$ and $\mathrm{NaOH}$. Sample undersized; diluted; $93 \%$ sample.

\section{Lu-2455. L:a Köpinge 19:1, A 292}

$$
\delta^{13} \mathrm{2780} \pm \mathbf{9 0}
$$

Charcoal (Alnus sp \& unid) from post hole at same site as Lu-2460, above. Archaeol estimate: Late Bronze or Early Iron Age. Comment: no pretreatment; sample undersized; diluted; $43 \%$ sample

\section{Lu-2459. L:a Köpinge 19:1, A 332}

$3350 \pm 100$

Unid. small charcoal particles from post hole (?) at same site as Lu2460, above. Archaeol estimate: Late Bronze or Early Iron Age. Comment: mild pretreatment with $\mathrm{NaOH}$ and $\mathrm{HC}$. Sample undersized; diluted; $37 \%$ sample.

Lu-2458. L:a Köpinge 19:1, A 335

$$
\begin{array}{r}
\mathbf{3 7 6 0} \pm \mathbf{6 0} \\
\delta^{13} C=-25.5 \% \text { o }
\end{array}
$$

Unid. small charcoal particles from soot pit at same site as Lu-2460, above. Archaeol estimate: Late Bronze Age or Early Iron Age. Comments: pretreated with $\mathrm{HCl}$ and $\mathrm{NaOH}$. (ST): older than expected from assoc archaeol finds.

\section{Lu-2462. L:a Köpinge 19:1, A 388}

$\mathbf{2 7 0 0} \pm \mathbf{9 0}$

Charcoal (Ouercus sp, Betula sp, Fraxinus sp, Corylus avellana, Acer sp, Alnus sp, \& Tilia sp) from post hole at same site as Lu-2460, above. Archaeol estimate: Late Bronze or Early Iron Age. Comment: no pretreatment; sample undersized; diluted; $41 \%$ sample.

\section{Lu-2454. L:a Köpinge 19:1, A 425}

$$
\begin{array}{r}
\mathbf{3 1 3 0} \pm \mathbf{7 0} \\
\delta^{13} C=-26.7 \% 0
\end{array}
$$

Charcoal (Corylus avellana, Alnus sp, Prunus sp, Pomoideae, \& unid.) from hearth at same site as Lu-2460, above. Archaeol estimate: Late Bronze or Early Iron Age. Comment: pretreated with $\mathrm{HCl}$ and $\mathrm{NaOH}$.

\section{Lu-2453. L:a Köpinge 19:1, A 581}

$$
\begin{array}{r}
\mathbf{2 7 8 0} \pm \mathbf{9 0} \\
\delta^{13} C=-27.3 \% \text { o }
\end{array}
$$

Charcoal (Alnus sp) from hearth at same site as Lu-2460, above. Archaeol estimate: Late Bronze or Early Iron Age. Comment: no pretreatment; sample undersized; diluted; $41 \%$ sample. 


\section{Lu-2457. L:a Köpinge 19:1, A 591}

$\mathbf{1 9 2 0} \pm \mathbf{5 0}$

$\delta^{13} \mathrm{C}=-26.8 \%$

Charcoal (Betula sp \& Alnus sp) from hearth at same site as Lu-2460, above. Archaeol estimate: Late Bronze or Early Iron Age. Comments: pretreated with $\mathrm{HCl}$ and $\mathrm{NaOH}$. (ST): somewhat later than expected.

\section{Lu-2452. L:a Köpinge 19:1, A 860}

$1690 \pm 50$

$$
\delta^{13} \mathrm{C}=-28.4 \%
$$

Charcoal (Alnus sp) from hearth at same site as Lu-2460, above. Archaeol estimate: Late Bronze or Early Iron Age. Comments: pretreated with $\mathrm{HCl}$ and $\mathrm{NaOH}$. (ST): later than expected from assoc archaeol finds.

Lu-2449. Köpinge 66:1, A 1

$$
\begin{array}{r}
\mathbf{2 4 8 0} \pm \mathbf{6 0} \\
\delta^{13} C=-25.4 \% 0
\end{array}
$$

Charcoal (Corylus avellana) from Construction No. 1 at Köpinge 66:1 $\left(55^{\circ} 28^{\prime} \mathrm{N}, 13^{\circ} 59^{\prime} \mathrm{E}\right)$. Comment: pretreated with $\mathrm{HCl}$ and $\mathrm{NaOH}$. Sample undersized; diluted; $89 \%$ sample.

\section{Lu-2456. Öja 1:1, A 7 \\ $\delta^{13} C=-25.3 \%$ \\ Charcoal (Pomoideae, Rhamnus cathartica, Alnus sp, \& Ulmus sp) from

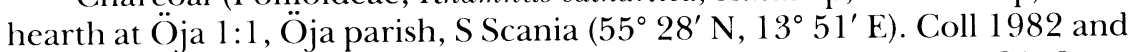 subm by $\mathrm{S}$ Tesch. Comment: mild pretreatment with $\mathrm{NaOH}$ and HC1. Sam- ple undersized; diluted; $86 \%$ sample.}

$\mathbf{1 9 8 0} \pm \mathbf{5 0}$

\section{Nymölla series (III)}

Charcoal from coastal settlement area (Middle Neolithic-Pitted Ware culture) at Nymölla $12^{38}$, Gualöv parish, NE Scania $\left(56^{\circ} 02^{\prime} \mathrm{N}, 14^{\circ} 28^{\prime} \mathrm{E}\right)$. Coll 1982 and subm by B Wyszomirska, Inst Archaeol, Univ Lund. For other dates from Nymölla, see R, 1982, v 24, p 210; 1984, v 26, p 407. Pretreated with $\mathrm{HCl}$ and $\mathrm{NaOH}$.

\section{Lu-2493. Nymölla 12 $2^{38}$, Sq L9}

$$
\begin{array}{r}
\mathbf{4 4 5 0} \pm \mathbf{6 0} \\
\delta^{13} \mathrm{C}=-25.0 \%
\end{array}
$$

Charcoal from occupation layer, Sq L9. Assoc with flint tools and Pitted Ware pottery indicating Middle Neolithic Age.

\section{Lu-2494. Nymölla $12^{38}, \mathrm{Sq} \mathrm{m9/1}$}

$3610 \pm 70$

$\delta^{13} \mathrm{C}=-26.0 \%$

Charcoal from occupation layer, $\mathrm{Sq} \mathrm{m} 9,+7.38 \mathrm{~m}$. Assoc with same artifact assemblage as Lu-2493, above. Comment: sample undersized; diluted; $70 \%$ sample.

\section{Lu-2495. Nymölla $12^{38}, \mathrm{Sq} \mathbf{m 9} / 2$}

$4360 \pm 60$

Charcoal from hearth, $\mathrm{Sq} \mathrm{m} 9,+7.46 \mathrm{~m}$. Assoc with same artifact assemblage as Lu-2493, above. 
Denmark

\section{Hareskovene series}

Charcoal from sites in Store and Lille Hareskov, NW of Copenhagen. Coll 1979 (Lu-2469) and 1984 and subm by E Laumann Jörgensen, Værlöse Mus, Værlöse. Dating is part of study of stones with incisions of sacral character (Laumann Jörgensen, 1980). For previous date from Store Hareskov, see R, 1982, v 24, p 211. All samples pretreated with HCl and $\mathrm{NaOH}$. No $\delta^{13} \mathrm{C}$ measurements available for this series. Standard deviation for dates increased accordingly.

\section{Lu-2467. Kærmysse-mosen, Sample I}

$600 \pm 50$

Charcoal from uppermost part of presumed cremation grave at Bøndernes Hegn Afd 199A, Kærmysse-mosen, Store Hareskov, 5km NNW of Gladsakse village $\left(55^{\circ} 46.5^{\prime} \mathrm{N}, 12^{\circ} 26^{\prime} \mathrm{E}\right)$. Sample from flint accumulation ca $20 \mathrm{~cm}$ below surface.

Lu-2468. Kærmysse-mosen, Sample II

$540 \pm 50$

Charcoal from basal part of presumed cremation grave. Sample from same flint accumulation as Lu-2467, above, but ca $40 \mathrm{~cm}$ below surface.

\section{Lu-2469. Hörgen-Fægyden}

$100 \pm 50$

Charcoal from stone slab in front of stone with sacral incisions at Hörgen-Fægyden, Afd $117 \phi s t$, Lille Hareskov, 6km NW of Gladsakse village $\left(55^{\circ} 46.2^{\prime} \mathrm{N}, 12^{\circ} 23^{\prime} \mathrm{E}\right)$.

General Comment: dates much younger than expected.

$$
\text { Bulgaria }
$$

\section{Lu-2409. Varna}

Small charcoal particles separated from soot and mineral soil from archaeol excavation in center of town of Varna, W coast of Black Sea, $\mathrm{F}$ Bulgaria $\left(43^{\circ} 10^{\prime} \mathrm{N}, 27^{\circ} 57^{\prime} \mathrm{E}\right)$. Coll Sept 1983 and subm by M Filipova, Natural Mus, Varna. Sample contained a few subfossil seeds of cereal. Assoc with animal bones and pottery indicating Archaic period (6th to 5 th century BC). Comment: mild pretreatment with $\mathrm{NaOH}$ and $\mathrm{HC}$. Sample undersized; diluted; $78 \%$ sample.

\section{REFERENCES}

Áskelsson, J, 1934, Kvartärgeologische Studien von Island: Geol Fören Stockholm Förh, v 56, p 596-618

Firbas, F, 1949, Spät- und nacheiszeitliche Waldgeschichte Mitteleuropas nördlich der Alpen, $\mathrm{I}$ : Jena, Fischer Verlag, $480 \mathrm{p}$.

Håkansson, S, 1977, University of Lund radiocarbon dates X: Radiocarbon, v 19, no. 3, p $424-441$

1979, Radiocarbon activity in submerged plants from various South Swedish lakes, in Berger, $\mathrm{R}$ and Suess, $\mathrm{H}$ E, eds, Radiocarbon dating, Internatl ${ }^{14} \mathrm{C}$ conf, 9 th, Proc: Berkeley, Univ California Press, p 433-443.

194-1982, University of Lund radiocarbon dates XV: Radiocarbon, v 24, no. 2, p 
Håkansson, S, 1983a, University of Lund radiocarbon dates XVI: Radiocarbon, v 25, no. 3, p $875-891$.

1983b, A reservoir age for the coastal waters of Iceland: Geol Fören Stockholm Förh, v 105, pt 1, p 64-67.

Forh, 1984, University of Lund radiocarbon dates XVII: Radiocarbon, v 26, no. 3, p $392-411$.

$141-164$

Ingólfsson, O, 1985, Late Weichselian glacial geology of the Lower Borgarfjördur Region, Western Iceland: a preliminary report: Arctic, v 38, no. 3, p 210-213.

Kjartansson, G, 1943, Arnesinga saga: Arnesingafélagid i Reykjavík (in Icelandic), 268 p.

Kjartansson, G, Thórarinsson, S and Einarsson, Th, 1964, $\mathrm{C}^{14}$ aldursákvardanir á sýnishornum vardandi islenska Kvarterjardfraedi: Nattúrufrædingurinn, v 34, p 97-145.

Larsson L, 1983, Skateholmsprojektet. Jägare-fiskare-bönder: Limhamniana (Malmö) 1983, p $7-40$

1984, Skateholmsprojektet. På spåren efter gravsedsförändringar, ceremoniplatser och tama rävar: Limhamniana (Malmö) 1984, p 49-84.

Laumann Jörgensen, E, 1980, Sakrale riller i sten. Upåagtede helleristninger: Hist Forening for Værlöse Kommune, Arsskr 1980, p 9-56.

Malmer, M P, 1962, Jungneolithische Studien: Acta Archaeol Lundensia, ser in $8^{\circ}$, no. 2, XXXVI +959 p.

Malmer, N, 1962, Studies on mire vegetation in Archaean area of southwestern Götaland, South Sweden. I. Vegetation and habitat conditions on the Åkhult mire: Opera Bot (Lund), v 7:1, $322 \mathrm{p}$.

Nilsson T, 1964, Standardpollendiagramme und $\mathrm{C}^{14}$-datierungen aus dem Ageröds Mosse im mittleren Schonen: Lunds Univ Årsskr, NF Avd 2, v 59, no. 7, p 1-52.

Norddahl, H, 1983, Late Quaternary stratigraphy of Fnjóskadalur, Central North Iceland, a study of sediments, ice-lake strandlines, glacial isostasy and ice-free areas: Thesis 12, Dept Quaternary Geol, Univ Lund, 78 p.

Olsson, I U, 1980, Content of ${ }^{14} \mathrm{C}$ in marine mammals from northern Europe, in Stuiver, $\mathrm{M}$ and Kra, R S, eds, Internatl ${ }^{14} \mathrm{C}$ conf, 10th, Proc: Radiocarbon, v 22, no. 3, p 662-675.

Rapp, A, 1984, Nivation hollows and glacial cirques in Söderåsen, Scania, South Sweden: Geog Annaler, v 66A(1-2), p 11-28.

Tesch, S, 1983, Ystad II. En omlandsstudie: Rapport Medeltidsstaden 45, Riksantikvarieämbetet och statens hist mus, Stockholm, $134 \mathrm{p}$. 